\title{
SOLAR-LIKE OSCILLATIONS IN LOW-LUMINOSITY RED GIANTS: FIRST RESULTS FROM KEPLER
}

\author{
T. R. Bedding ${ }^{1}$, D. Huber ${ }^{1}$, D. Stelloo ${ }^{1}$, Y. P. Elsworth ${ }^{2}$, S. Hekker ${ }^{2}$, T. Kallinger ${ }^{3,4}$, S. Mathur $^{5}$, B. Mosser ${ }^{6}$, \\ H. L. Preston ${ }^{7,8}$, J. Ballot ${ }^{9}$, C. Barban ${ }^{6}$, A. M. Broomhall ${ }^{2}$, D. L. Buzasi ${ }^{7}$, W. J. Chaplin ${ }^{2}$, R. A. García ${ }^{10}$, \\ M. Gruberbauer ${ }^{11}$, S. J. Hale ${ }^{2}$, J. De Ridder ${ }^{12}$, S. Frandsen ${ }^{13}$, W. J. Borucki ${ }^{14}$, T. Brown ${ }^{15}$, J. Christensen-DalsgaArd $^{13}$, \\ R. L. Gilliland ${ }^{16}$, J. M. Jenkins ${ }^{17}$, H. KJeldsen ${ }^{13}$, D. KoCH $^{14}$, K. BelKaCem ${ }^{18}$, L. Bildsten ${ }^{19}$, H. BruntT ${ }^{6,13}$, \\ T. L. Campante ${ }^{13,20}$, S. Deheuvels ${ }^{6}$, A. Derekas ${ }^{1,21}$, M.-A. Dupret ${ }^{18}$, M.-J. Goupil ${ }^{6}$, A. Hatzes ${ }^{22}$, G. HoudeK $^{4}$,

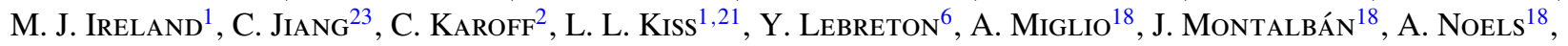 \\ I. W. Roxburgh ${ }^{24}$, V. SAngaralingam ${ }^{2}$, I. R. Stevens ${ }^{2}$, M. D. Suran ${ }^{25}$, N. J. TARrant ${ }^{2}$, And A. Weiss ${ }^{26}$ \\ ${ }^{1}$ Sydney Institute for Astronomy (SIfA), School of Physics, University of Sydney, NSW 2006, Australia; bedding @ physics.usyd.edu.au \\ ${ }^{2}$ School of Physics and Astronomy, University of Birmingham, Birmingham B15 2TT, UK \\ ${ }^{3}$ Department of Physics and Astronomy, University of British Columbia, Vancouver, Canada \\ ${ }^{4}$ Institute of Astronomy, University of Vienna, 1180 Vienna, Austria \\ ${ }^{5}$ Indian Institute of Astrophysics, Koramangala, Bangalore 560034, India \\ ${ }^{6}$ LESIA, CNRS, Université Pierre et Marie Curie, Université Denis, Diderot, Observatoire de Paris, 92195 Meudon Cedex, France \\ ${ }^{7}$ Eureka Scientific, 2452 Delmer Street Suite 100, Oakland, CA 94602-3017, USA \\ ${ }^{8}$ Department of Mathematical Sciences, University of South Africa, Box 392 UNISA 0003, South Africa \\ ${ }^{9}$ Laboratoire d'Astrophysique de Toulouse-Tarbes Université de Toulouse, CNRS 14 av E. Belin 31400 Toulouse, France \\ ${ }^{10}$ Laboratoire AIM, CEA/DSM-CNRS, Université Paris 7 Diderot, IRFU/SAp, Centre de Saclay, 91191, Gif-sur-Yvette, France \\ ${ }^{11}$ Department of Astronomy and Physics, Saint Mary's University, Halifax, NS B3H 3C3, Canada \\ ${ }^{12}$ Instituut voor Sterrenkunde, K.U.Leuven, Belgium \\ ${ }^{13}$ Danish AsteroSeismology Centre (DASC), Department of Physics and Astronomy, Aarhus University, DK-8000 Aarhus C, Denmark \\ ${ }^{14}$ NASA Ames Research Center, MS 244-30, Moffett Field, CA 94035, USA \\ ${ }^{15}$ Las Cumbres Observatory Global Telescope, Goleta, CA 93117, USA \\ ${ }^{16}$ Space Telescope Science Institute, 3700 San Martin Drive, Baltimore, MD 21218, USA \\ 17 SETI Institute/NASA Ames Research Center, MS 244-30, Moffett Field, CA 94035, USA \\ ${ }^{18}$ Institut d'Astrophysique et de Géophysique de l'Université de Liège, Allée du 6 Août 17-B 4000 Liège, Belgium \\ ${ }^{19}$ Kavli Institute for Theoretical Physics and Department of Physics, University of California, Santa Barbara, CA 93106, USA \\ ${ }^{20}$ Centro de Astrofísica da Universidade do Porto, Rua das Estrelas, 4150-762 Porto, Portugal \\ ${ }^{21}$ Konkoly Observatory of the Hungarian Academy of Sciences, H-1525 Budapest, P.O. Box 67, Hungary \\ 22 Thueringer Landessternwarte Tautenburg, Sternwarte 5, D-07778, Tautenburg, Germany \\ ${ }^{23}$ Department of Astronomy, Beijing Normal University, China \\ ${ }^{24}$ Queen Mary University of London, Mile End Road, London E1 4NS, UK \\ ${ }^{25}$ Astronomical Institute of the Romanian Academy, Str. Cutitul de Argint, 5, RO 40557,Bucharest, Romania \\ ${ }^{26}$ Max-Planck-Institut für Astrophysik, Karl-Schwarzschild-Str. 1, 85748 Garching, Germany \\ Received 2009 November 13; accepted 2009 December 21; published 2010 March 31
}

\begin{abstract}
We have measured solar-like oscillations in red giants using time-series photometry from the first 34 days of science operations of the Kepler Mission. The light curves, obtained with 30 minute sampling, reveal clear oscillations in a large sample of $\mathrm{G}$ and $\mathrm{K}$ giants, extending in luminosity from the red clump down to the bottom of the giant branch. We confirm a strong correlation between the large separation of the oscillations $(\Delta v)$ and the frequency of maximum power $\left(v_{\max }\right)$. We focus on a sample of 50 low-luminosity stars $\left(v_{\max }>100 \mu \mathrm{Hz}, L \lesssim 30 L_{\odot}\right)$ having high signal-to-noise ratios and showing the unambiguous signature of solar-like oscillations. These are $\mathrm{H}$-shellburning stars, whose oscillations should be valuable for testing models of stellar evolution and for constraining the star formation rate in the local disk. We use a new technique to compare stars on a single échelle diagram by scaling their frequencies and find well-defined ridges corresponding to radial and non-radial oscillations, including clear evidence for modes with angular degree $l=3$. Measuring the small separation between $l=0$ and $l=2$ allows us to plot the so-called C-D diagram of $\delta v_{02}$ versus $\Delta v$. The small separation $\delta v_{01}$ of $l=1$ from the midpoint of adjacent $l=0$ modes is negative, contrary to the Sun and solar-type stars. The ridge for $l=1$ is notably broadened, which we attribute to mixed modes, confirming theoretical predictions for low-luminosity giants. Overall, the results demonstrate the tremendous potential of Kepler data for asteroseismology of red giants.
\end{abstract}

Key words: stars: oscillations

Online-only material: color figure

\section{INTRODUCTION}

Studying solar-like oscillations is a powerful way to probe the interiors of stars (e.g., Brown \& Gilliland 1994; ChristensenDalsgaard 2004). Oscillations in main-sequence stars and subgiants have been measured using ground-based spectroscopy (for a recent review, see Aerts et al. 2008) and more recently by space missions such as CoRoT (e.g., Michel et al. 2008).
Red giants oscillate at lower frequencies and so demand long and preferably uninterrupted time series to resolve their oscillations.

The first indications of solar-like oscillations in $\mathrm{G}$ and $\mathrm{K}$ giants were based on ground-based observations in radial velocity (see Merline 1999 and references therein; Frandsen et al. 2002; De Ridder et al. 2006) and photometry (Stello et al. 2007), and on space-based photometry from the Hubble Space Telescope 
(HST; Edmonds \& Gilliland 1996; Gilliland 2008; Stello \& Gilliland 2009), WIRE (Buzasi et al. 2000; Retter et al. 2003; Stello et al. 2008), Microvariability and Oscillations of Stars (MOST; Barban et al. 2007; Kallinger et al. 2008a, 2008b), and Solar Mass Ejection Imager (SMEI) (Tarrant et al. 2007). A major breakthrough came from observations over about 150 days with the CoRoT space telescope, which produced clear detections in numerous stars of both radial and non-radial oscillations in the frequency range $10-100 \mu \mathrm{Hz}$ (De Ridder et al. 2009; Hekker et al. 2009; Carrier et al. 2010). This increased the number of known pulsating $\mathrm{G}$ and $\mathrm{K}$ giants to nearly 800 , and enabled the first systematic study of oscillations in a large population of red clump stars (Hekker et al. 2009; Miglio et al. 2009; Kallinger et al. 2010). Note that the red clump comprises core He-burning stars and is the metal-rich counterpart to the horizontal branch.

Detecting oscillations in lower-luminosity red giants, which are $\mathrm{H}$-shell-burning stars at the base of the giant branch, is very desirable for testing models of stellar evolution and also for constraining the star formation rate in the local disk (Miglio et al. 2009). However, such detections are even more challenging than for clump stars because the amplitudes are substantially lower. In addition, the oscillation frequencies are higher and are comparable to the orbital frequency of a satellite in low-Earth orbit. These factors made it difficult for CoRoT to achieve such detections.

In this Letter, we present data from the asteroseismology program of the NASA Kepler Mission (Gilliland et al. 2010) that reveal clear oscillations in a large sample of red giants, extending in luminosity from the red clump down to the bottom of the giant branch.

\section{GLOBAL OSCILLATION PARAMETERS}

The observations were obtained over the first 34 days of science operations of the Kepler Mission (Q1 data). We analyzed light curves having 29.4-minute sampling (long-cadence mode) for about 1500 stars listed as red giants in the Kepler Input Catalog (KIC; Latham et al. 2005). About 20\% of the Q1 light curves showed large variations ( $>1 \%$ ) on long timescales, mostly due to $\mathrm{M}$ giant pulsations, and are not analyzed here. ${ }^{27}$ For the remaining sample, we used the light curves to measure the global oscillation parameters, most notably the frequency of maximum power $\left(v_{\max }\right)$ and the mean large frequency separation $(\Delta v)$. We were able to find a power excess in $\sim 1000$ stars (see also Figure 5 in Gilliland et al. 2010) and measure the large separation for $\sim 700$ stars. In this Letter, we focus on the lowluminosity red giants (corresponding to $v_{\max }>100 \mu \mathrm{Hz}$ ) for which, as discussed in the introduction, CoRoT results have not yet been reported. As discussed by Miglio et al. (2009), such stars generally have luminosities below about $30 L_{\odot}$.

Figure 1 shows the measured values of $\Delta v$ and $v_{\max }$ for these stars. The symbols show results obtained using the pipeline described by Huber et al. (2009), who also described the method used to fit and subtract the background. Similar results were found using pipelines developed by four other groups (Hekker et al. 2010b; Mosser \& Appourchaux 2009; Kallinger et al. 2010; Mathur et al. 2010), and the dotted lines in Figure 1 show the region inside which $\sim 80 \%$ of those measurements lay. A detailed comparison of the results from the different pipelines is ongoing.

\footnotetext{
27 One of these was subsequently found to be an oscillating red giant in an
} eclipsing binary system (Hekker et al. 2010a).

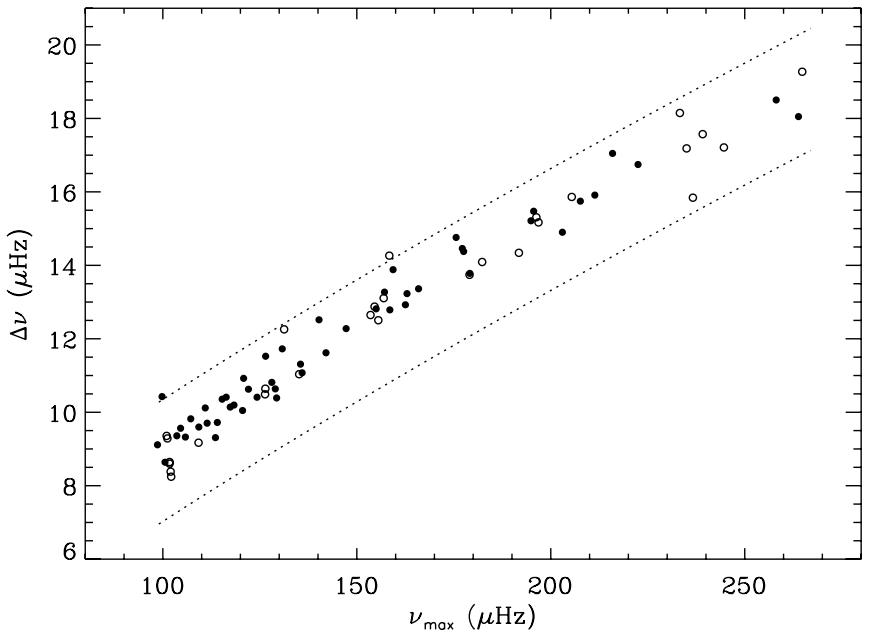

Figure 1. Large frequency separation vs. frequency of maximum power for 78 low-luminosity red giants. Filled symbols indicate the 50 stars that were selected for more detailed analysis. The dotted lines enclose the region containing $\sim 80 \%$ of measurements by the five pipelines (see the text).

The tight correlation in Figure 1 between $\Delta v$ and $v_{\max }$ was discussed by Hekker et al. (2009) and Stello et al. (2009), and is seen here to extend to red giants with much higher $v_{\max }$. This and other correlations between global oscillation parameters, and their connection to fundamental stellar parameters, will be discussed in future papers.

\section{RESULTS}

\subsection{Frequency Analysis}

We now consider a subset of 50 of the low-luminosity red giants for more detailed analysis, chosen as having the best signal-to-noise ratios. These stars are indicated in Figure 1 with filled symbols. Figure 2 (left panel) shows power spectra for six of them, spanning the full range of $v_{\max }$ being considered. Each power spectrum shows a regular series of peaks, which is the clear signature of solar-like oscillations.

Solar-like oscillations are $p$ modes with high order and low degree, and the observed frequencies in main-sequence stars are reasonably well described by the following relation:

$$
v_{n, l} \approx \Delta v\left(n+\frac{1}{2} l+\epsilon\right)-l(l+1) D_{0} .
$$

Here, $n$ (the radial order) and $l$ (the angular degree) are integers. The form of Equation (1) is motivated by theoretical calculations: for relatively unevolved stars, an asymptotic expansion (Tassoul 1980; Gough 1986) shows that $\Delta v$ is approximately the inverse of the sound travel time across the star, while $D_{0}$ is sensitive to the sound speed gradient near the core, and $\epsilon$ is sensitive to the surface layers.

We conventionally define three small frequency separations: $\delta v_{02}$ is the spacing between adjacent modes with $l=0$ and $l=2, \delta v_{13}$ is the spacing between adjacent modes with $l=1$ and $l=3$, and $\delta v_{01}$ is the amount by which $l=1$ modes are offset from the midpoint between the $l=0$ modes on either side. If Equation (1) holds, then it follows that $\delta v_{02}=6 D_{0}$, $\delta v_{13}=10 D_{0}$, and $\delta v_{01}=2 D_{0}$. We shall use Equation (1) as a guide to the analysis of the observed frequencies, although its physical interpretation may be open to question in the case of red giants.

To determine oscillation frequencies for each of the 50 stars in our subset, we needed to locate the highest peaks in 


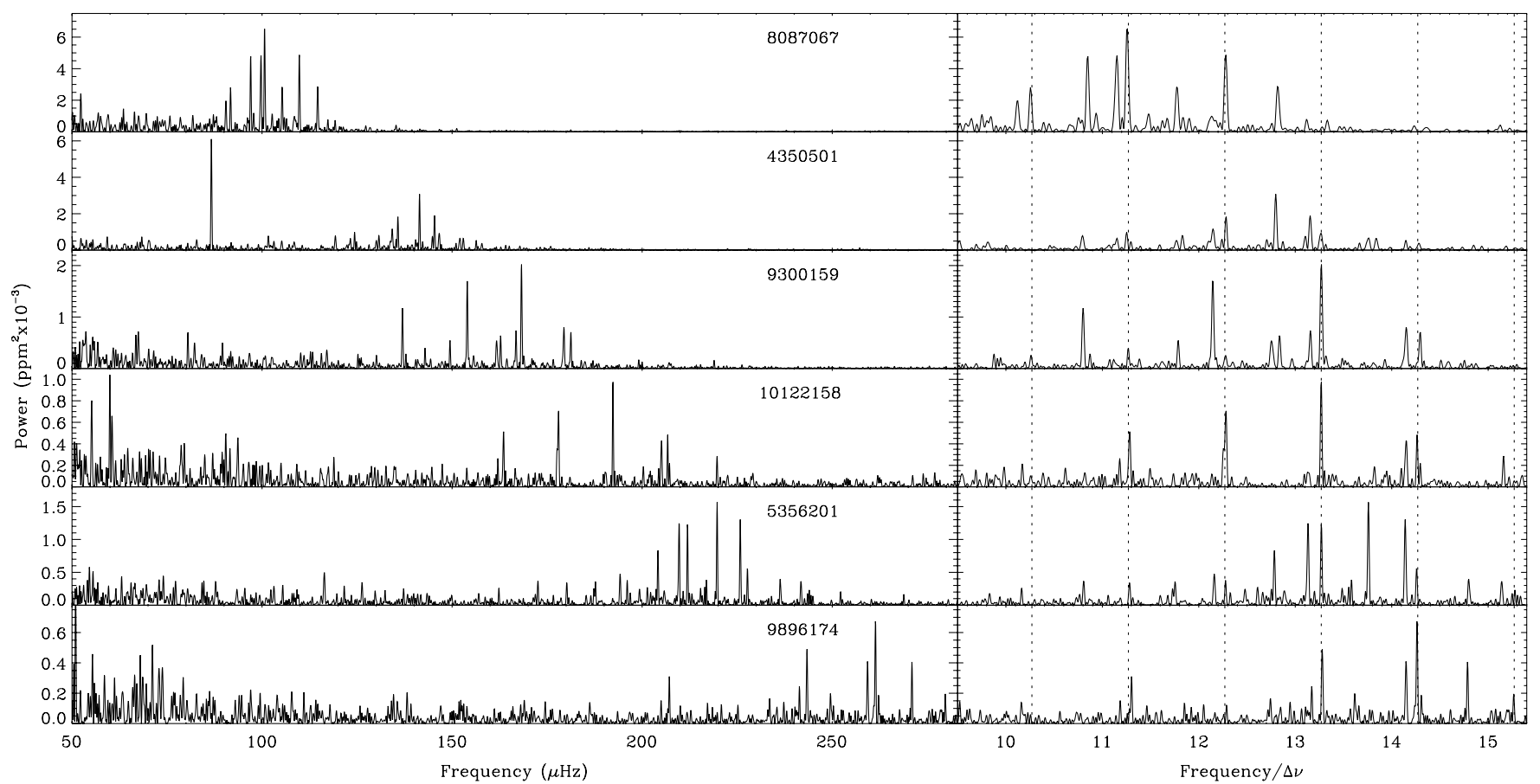

Figure 2. Left: power spectra of six representative low-luminosity red giants. Right: the same power spectra plotted against scaled frequency (see Section 3.2). The dotted lines are equally spaced, having unit separation and being aligned with the $l=0$ modes. Stars are labeled with identification numbers from the KIC (Latham et al. 2005).

the power spectra. We did this using conventional iterative sine-wave fitting, sometimes called pre-whitening or CLEAN. This involves finding the highest peak in the power spectrum, subtracting the corresponding sinusoidal variation from the time series and then re-calculating the power spectrum in an iterative process. We retained only those peaks that lay in the frequency range $v_{\max } \pm 5 \Delta v$ and had heights greater than 4.5 times the fitted background level (in amplitude).

Damping of solar-like oscillations causes the observed power from each mode to be spread into multiple peaks under a Lorentzian envelope. If the length of the observations is significantly greater than the typical mode lifetime, one therefore expects several peaks to be extracted for each mode. In fact, the modes are only barely resolved (see Section 3.3) and so we expect iterative sine-wave fitting to give good results.

\subsection{Semi-scaled Échelle Diagram}

A powerful method to study the mode frequencies of solarlike oscillations is to plot them modulo the large separation, in a so-called échelle diagram (Grec et al. 1983). We have displayed the frequencies of all 50 stars in a single échelle diagram by using the scaling technique described by Bedding \& Kjeldsen (2010), in an effort to carry out "ensemble asteroseismology." The result is shown in Figure 3(a). To make this diagram, we first divided the frequencies of each star by $\Delta v$ (that is, scaling them to have a large separation of unity). We used the échelle diagram to identify the radial modes and then fine-tuned the scaling factor $(\Delta \nu)$ by up to a few percent to align them on a single line in the échelle diagram, shown as a solid line in Figure 3(a). As discussed by Bedding \& Kjeldsen (2010, their Method 2), this equates to assigning all stars the same value of $\epsilon$. The fact that all the stars can be aligned indicates this is a valid approximation. This alignment is also clearly seen in the right-hand panel of Figure 2, which shows power spectra plotted against scaled frequencies (i.e., divided by $\Delta v$ ).
The échelle diagram in Figure 3(a) differs from those shown by Bedding \& Kjeldsen (2010) in one important respect: only the horizontal axis has been scaled. In the vertical direction, we have plotted the original frequencies without scaling, which spreads out the points and allows us to look for variations with $v_{\max }$. Such a plot might be called a semi-scaled échelle diagram.

\subsection{Collapsed Échelle Diagram and Mode Lifetimes}

We can collapse the échelle diagram in the vertical direction in order to define the ridges more clearly. Figure 3(b) shows this using a simple histogram of the peaks in Figure 3(a), and the ridges are clearly visible. Rather than counting the number of peaks above a threshold, another approach is to sum the total power. To do this, we restricted each backgroundcorrected power spectrum to the same frequency range as before $\left(v_{\max } \pm 5 \Delta v\right)$, then divided the frequency scale by the large separation (using the same fine-tuned value as above) and folded the spectrum modulo unit spacing. These folded spectra were summed for all 50 stars and then smoothed slightly (with a boxcar of width $0.01 \Delta v$ ) to give the result shown in Figure 3(c).

The clarity of the ridges in Figure 3 allows us to make some statements about mode lifetimes. As mentioned in Section 3.1, damping causes each mode to be spread into multiple peaks under a Lorentzian envelope with a full-width at half maximum (FWHM) of $1 /(\pi \tau)$, where $\tau$ is the mode lifetime. There has been considerable discussion of the lifetimes of red giant oscillations, particularly regarding whether the modes are sufficiently long-lived to allow their frequencies to be measured with sufficient accuracy to be useful for asteroseismology (Houdek \& Gough 2002; Stello et al. 2006; Barban et al. 2007; Dupret et al. 2009). The CoRoT results have clearly dismissed this worry for red clump stars (De Ridder et al. 2009; Carrier et al. 2010), and our Kepler data do the same for the low-luminosity stars. This is excellent news for asteroseismology. 


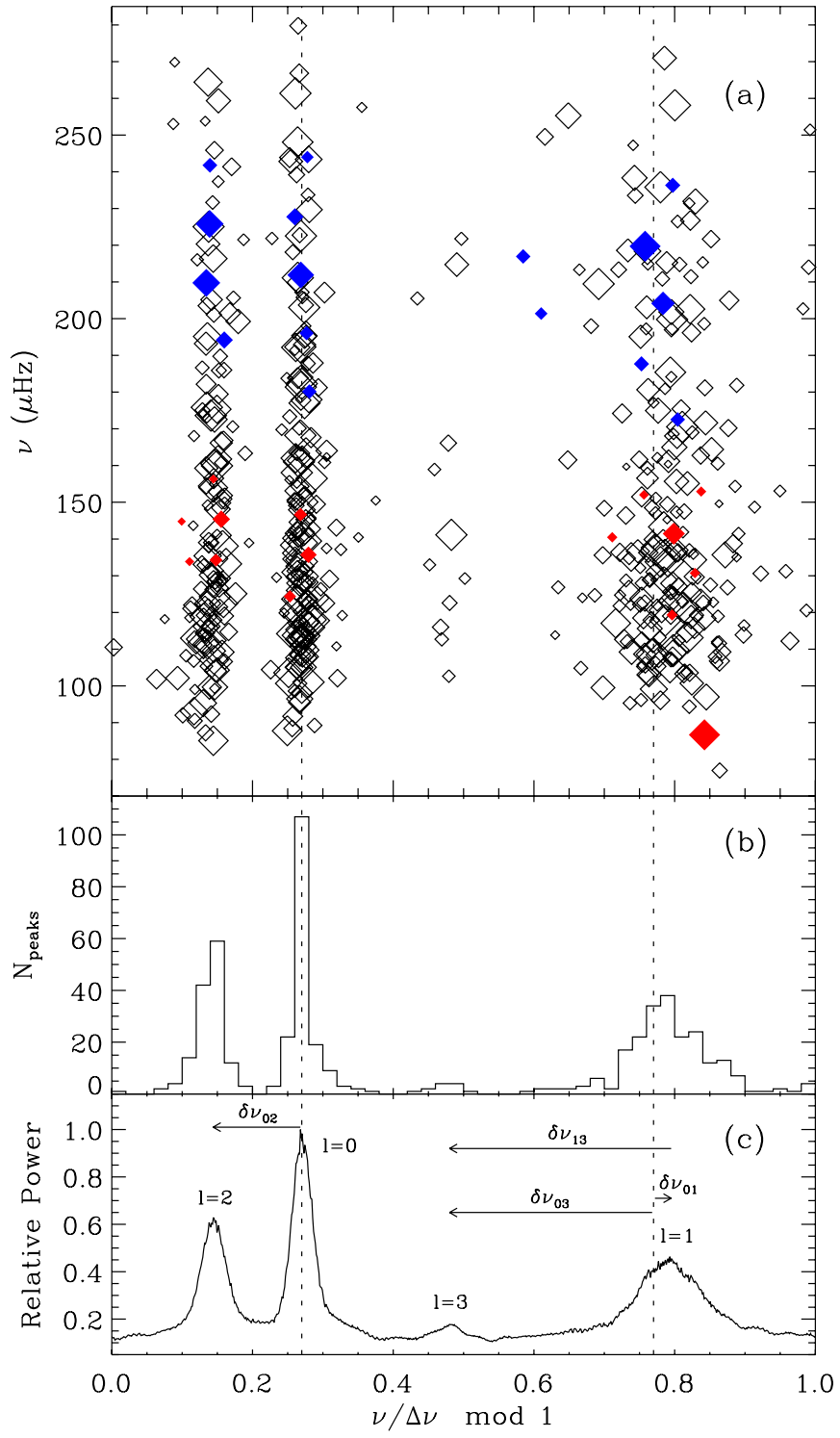

Figure 3. (a) Semi-scaled échelle diagram for the sample of 50 low-luminosity red giants (see Section 3.2). Symbols indicate extracted frequencies, with symbol sizes proportional to amplitude. Filled blue and red symbols are frequencies of the stars KIC 5356201 and KIC 4350501, respectively (see Figure 2 and Section 3.5). The value of $\Delta v$ for each star was fine-tuned in order to align the $l=0$ modes on the left-most vertical dotted line. The other dotted line lies exactly 0.5 away, marking the midpoint between $l=0$ modes. (b) Histogram of the points shown in panel (a). (c) Folded and scaled power spectra, collapsed over all stars in the sample and smoothed slightly (see Section 3.2). The arrows show the definitions of the small separations $\delta v_{01}, \delta v_{02}, \delta v_{03}$, and $\delta v_{13}$, with the sign convention that left-pointing arrows correspond to positive separations (see Section 3.1).

(A color version of this figure is available in the online journal.)

Figure 3 was constructed by aligning the radial modes on the vertical solid line. However, the resulting width of the $l=0$ ridge is not zero, which arises from at least three factors: (1) the resolution limit set by the duration of the observations (34 days), which leads to peaks in the power spectrum having FWHM $0.3 \mu \mathrm{Hz} ;$ (2) variations in $\Delta v$ with frequency in individual stars over the range being considered (curvature in the échelle diagram); and (3) broadening of power due to finite mode lifetimes (see the above). The observed narrowness of the $l=0$ ridge sets upper limits on the second and third of these effects. Here, as discussed above, we are particularly interested in the mode lifetimes.

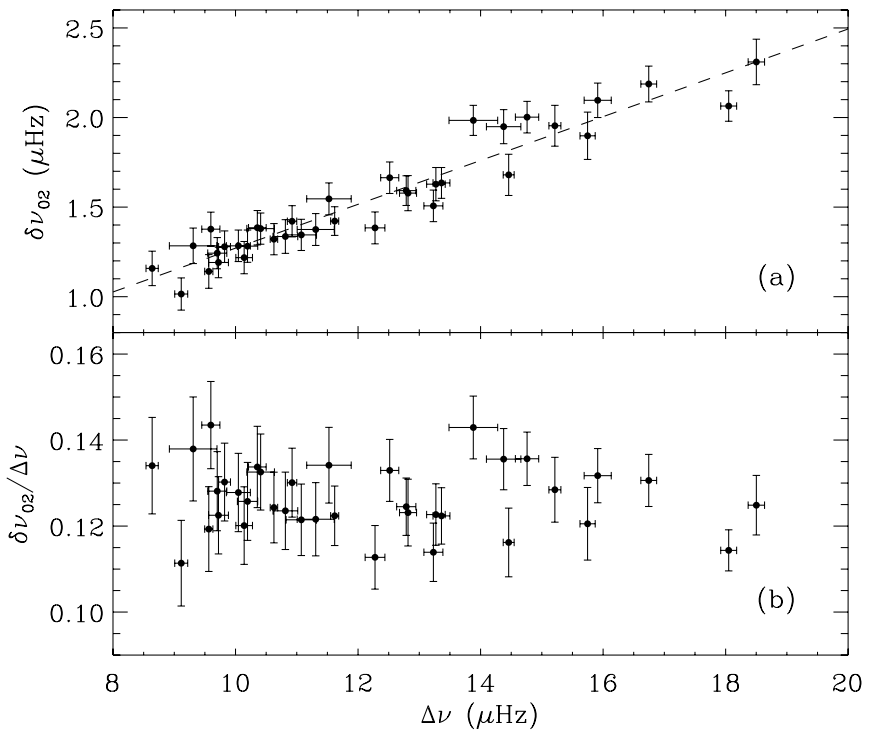

Figure 4. (a) So-called C-D diagram (Christensen-Dalsgaard 1988) of $\delta v_{02}$ vs. $\Delta v$ for the 38 stars in our sample for which both quantities were reliably determined. The dashed line shows a linear fit. (b) The ratio $\delta v_{02} / \Delta v$ versus $\Delta v$ for the same stars, to better show the scatter about the trend.

The FWHM of the $l=0$ peak in Figure 3(c), found by subtracting the background and fitting a Lorentzian, is $0.033 \Delta v$. This sets an upper limit on the intrinsic line widths of these modes. However, so far our measurement is in terms of $\Delta v$, which varies over the sample. The median $\Delta v$ is $11.5 \mu \mathrm{Hz}$, in which case the measured width equates to a lower limit on the mode lifetimes of about 10 days. To investigate further, we divided the sample into four bins according to $\Delta v$ and formed the collapsed échelle diagram for each subset, as before. We found the widths of the $l=0$ ridges in all four cases to be about the same in absolute terms $(\sim 0.4 \mu \mathrm{Hz})$, which is only slightly greater than the FWHM set by the resolution of the data (see the above). We conclude that the mode lifetimes in these red giants is at least 10 days, possibly much greater.

\subsection{Small Separations and the C-D Diagram}

The $l=2$ ridge is closely parallel to the $l=0$ ridge, implying that $\delta v_{02}$ is a nearly constant fraction of $\Delta v$ for the stars in our sample, with $\delta v_{02} \approx 0.125 \Delta v$ (Figure 3(c)). This frequency scaling indicates that, to a good approximation, the stars are homologous.

We were able to measure the mean small separation $\delta v_{02}$ for 38 stars in our sample. Figure 4(a) shows the results in a socalled C-D diagram (Christensen-Dalsgaard 1988), which plots $\delta v_{02}$ versus $\Delta v$. The dashed line in Figure 4(a) shows a linear fit, which has parameters

$$
\delta v_{02}=(0.122 \pm 0.006) \Delta v+(0.05 \pm 0.08) \mu \mathrm{Hz} .
$$

The spread of points about this relation presumably reflects a spread in stellar masses, and is better seen by plotting the ratio of the two separations, as shown in Figure 4(b) (see Roxburgh \& Vorontsov 2003; Otí Floranes et al. 2005; Mazumdar 2005).

The dotted line in Figure 3 marks the midpoint between $l=0$ modes. Interestingly, the $l=1$ ridge is centered slightly to the right of this line. This indicates that the average small separation $\delta v_{01}$ in these red giants is negative, whereas it is positive in the Sun and the handful of other main-sequence stars for which it has been measured: $\alpha$ Cen A (Bedding et al. 2004), $\alpha$ Cen B 

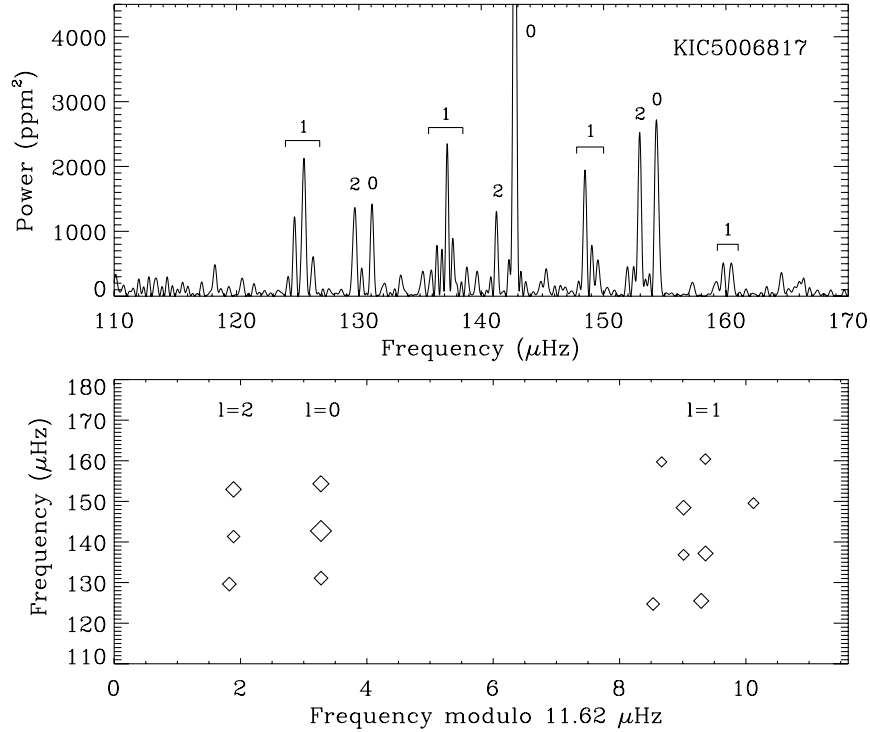

Figure 5. Power spectrum and the échelle diagram for the star KIC 5006817, illustrating multiple $l=1$ peaks per order. Note that the highest peak in the power spectrum extends beyond the plot limits (to $8400 \mathrm{ppm}^{2}$ ).

(Kjeldsen et al. 2005), $\gamma$ Pav (Mosser et al. 2008), and $\tau$ Cet (Teixeira et al. 2009). A negative value for $\delta v_{01}$ also indicates that Equation (1) is not satisfied in detail for these red giants, a point already noted for the G8 giant HR 7349 by Carrier et al. (2010), based on CoRoT observations.

\subsection{Mixed Modes with $l=1$}

The $l=1$ ridge in Figure 3 is notably broader than the others. We do not attribute this to shorter mode lifetimes, but rather to the presence of mixed modes whose frequencies are shifted by avoided crossings. Mixed modes occur in evolved stars and have the characteristics of $p$ modes in the envelope of the star and of $g$ modes in the interior (e.g., Aizenman et al. 1977). A typical example is the star KIC 5356201 (see Figure 2 and filled blue symbols in Figure 3(a)). The $l=0$ and 2 modes are quite close to their respective ridges, whereas there is much more scatter about $l=1$. A more extreme example is the star KIC 4350501 (see Figure 2 and filled red symbols in Figure 3(a)), which shows a particularly high peak at $87 \mu \mathrm{Hz}$, well below $v_{\max }$. The proximity of this peak to the $l=1$ ridge indicates that it is likely to be a mixed mode. A similar situation is seen in the F5 subgiant Procyon, for which ground-based velocity observations show a narrow peak well below $v_{\max }$ that lies close to the $l=1$ ridge in the échelle diagram (T. R. Bedding et al. 2010, in preparation). The large peak height can be explained by the fact that mixed modes are expected to have longer lifetimes (smaller line widths) than pure $p$ modes because they have larger mode inertias (e.g., Christensen-Dalsgaard 2004).

Another good example is KIC 5006817, shown in Figure 5, where we clearly see multiple $l=1$ peaks in each order (see also KIC $9904059=$ KOI 145 in Figure 1 of Gilliland et al. 2010). Each of these clusters of peaks is reminiscent of a single mode that is heavily damped (see Section 3.3). However, as mentioned above, mixed modes are expected to have longer lifetimes than pure $p$ modes and hence to produce narrower-not broader-peaks. Indeed, this phenomenon of multiple $l=1$ peaks is probably what led previous authors to report shortmode lifetimes in red giants (see Section 3.3). Guided by the theoretical work of Dupret et al. (2009), we instead interpret the observed properties of the $l=1$ ridge in terms of mixed modes.
Dupret et al. (2009) discussed theoretical amplitudes of solar-like oscillations for a range of models on the red giant branch. Their models show mostly regular frequency patterns for evolved giants $\left(v_{\max }<80 \mu \mathrm{Hz}\right.$ ), but predict more complex spectra for low-luminosity giants. This is mainly explained by the less efficient radiative damping in the cores of these stars, leading to a stronger interaction of $p$-mode and $g$-mode cavities, and by the less efficient trapping due to the smaller density contrast between the core and the envelope. As a consequence, more non-radial modes could be observed than only those trapped in the envelope. This is particularly expected to affect $l=1$ modes because the evanescent region just above the $\mathrm{H}$ burning shell is smaller for these modes (see also Dziembowski et al. 2001; Christensen-Dalsgaard 2004). Our observation of a broadened $l=1$ ridge with a small cluster of modes per order supports the theoretical predictions by Dupret et al. (2009). Observations of frequency spectra in a sample of higher luminosity red giants would be a desirable follow-up study.

\subsection{Detection of $l=3$ Modes}

In addition to ridges corresponding to $l=0,1$ and 2, we see clear evidence in Figure 3 for modes with $l=3$. The detection of these modes with photometry is difficult because of the very low amplitudes that result from geometric cancellation. The only previous report of solar-like oscillations with $l=3$ from photometry (except for the Sun) is a probable detection using CoRoT in the G0 main-sequence star HD 49385 (Deheuvels et al. 2009). The ability to detect $l=3$ modes from Kepler observations of red giants is a significant bonus.

In Figures 3(a) and (b) there are 12 peaks that fall along the ridge that we identify with $l=3$, and these arise from 10 stars in the sample of 50. These are presumably peaks that, due to the stochastic nature of the excitation, happen to rise above our detection threshold in these observations. A more objective approach is to sum the power from all stars, as shown in Figure 3(c), and we again see clear evidence for the $l=3$ ridge.

How does the position of the $l=3$ ridge compare with expectations? As mentioned in Section 3.1, it is conventional to measure $l=3$ relative to $l=1$, via the small separation $\delta v_{13}$. However, given that the position of $l=1$ for these red giants is anomalous, it seems sensible to instead measure $l=3$ relative to $l=0$. We therefore suggest using a new small separation, $\delta v_{03}$, defined (by analogy to $\delta v_{01}$ ) as the amount by which the $l=3$ modes are offset from the midpoint between the $l=0$ modes on either side. If follows that $\delta v_{03}=\delta v_{01}+\delta v_{13}$. From Figure 3(c) we see that $\delta v_{03} \approx 0.28 \Delta \nu$. If the asymptotic relation held (Equation (1)), we would expect $\delta v_{03}=12 D_{0}$. In that case, the ratio between $\delta v_{03}$ and $\delta v_{02}$ would be 2.0, whereas we observe a ratio of 2.2. These results should provide valuable tests for theoretical models.

\section{CONCLUSIONS}

These results, based on the analysis of only 34 days of data, show the tremendous potential of Kepler long-cadence data for the study of solar-like oscillations in red giants. As more data become available, the longer time series will allow detailed studies of stars covering the whole range of evolutionary states along the red giant branch and provide stringent observational tests for theories of stellar structure and evolution in this part of the H-R diagram. 
We gratefully acknowledge the entire Kepler team, whose outstanding efforts have made these results possible. Funding for this Discovery mission is provided by NASA's Science Mission Directorate. Grants were received from the European Research Council (ERC/FP7/2007-2013 n²27224, PROSPERITY), and K. U. Leuven (GOA/2008/04).

Facilities: Kepler

\section{REFERENCES}

Aerts, C., Christensen-Dalsgaard, J., Cunha, M., \& Kurtz, D. W. 2008, Sol Phys., 251, 3

Aizenman, M., Smeyers, P., \& Weigert, A. 1977, A\&A, 58, 41

Barban, C., et al. 2007, A\&A, 468, 1033

Bedding, T. R., \& Kjeldsen, H. 2010, Comm. Asteroseismology, 161, 3

Bedding, T. R., Kjeldsen, H., Butler, R. P., McCarthy, C., Marcy, G. W., O’Toole, S. J., Tinney, C. G., \& Wright, J. T. 2004, ApJ, 614, 380

Bedding, T. R., et al. 2010, ApJ, in press (arXiv:1003.0052)

Brown, T. M., \& Gilliland, R. L. 1994, ARA\&A, 32, 37

Buzasi, D., Catanzarite, J., Laher, R., Conrow, T., Shupe, D., Gautier, III, T. N., Kreidl, T., \& Everett, D. 2000, ApJ, 532, L133

Carrier, F., et al. 2010, A\&A, 509, 73

Christensen-Dalsgaard, J. 1988, in Proc. IAU Symp. 123, Advances in Helio- and Asteroseismology, ed. J. Christensen-Dalsgaard \& S. Frandsen (Dordrecht: Kluwer), 295

Christensen-Dalsgaard, J. 2004, Sol. Phys., 220, 137

Deheuvels, S., et al. 2009, A\&A, submitted

De Ridder, J., Barban, C., Carrier, F., Mazumdar, A., Eggenberger, P., Aerts, C., Deruyter, S., \& Vanautgaerden, J. 2006, A\&A, 448, 689

De Ridder, J., et al. 2009, Nature, 459, 398

Dupret, M., et al. 2009, A\&A, 506, 57

Dziembowski, W. A., Gough, D. O., Houdek, G., \& Sienkiewicz, R. 2001, MNRAS, 328, 601

Edmonds, P. D., \& Gilliland, R. L. 1996, ApJ, 464, L157

Frandsen, S., et al. 2002, A\&A, 394, L5

Gilliland, R. L. 2008, AJ, 136, 566

Gilliland, R. L., et al. 2010, PASP, 122, 131
Gough, D. O. 1986, in Hydrodynamic and Magnetodynamic Problems in the Sun and Stars, ed. Y. Osaki (Tokyo: Univ. Tokyo Press), 117

Grec, G., Fossat, E., \& Pomerantz, M. A. 1983, Sol. Phys., 82, 55

Hekker, S., et al. 2009, A\&A, 506, 465

Hekker, S., et al. 2010a, ApJ, 713, L187

Hekker, S., et al. 2010b, MNRAS, in press (arXiv:0911.2612)

Houdek, G., \& Gough, D. O. 2002, MNRAS, 336, L65

Huber, D., Stello, D., Bedding, T. R., Chaplin, W. J., Arentoft, T., Quirion, P., \& Kjeldsen, H. 2009, Commun. Asteroseismol., in press (arXiv:0910.2764)

Kallinger, T., et al. 2008a, Commun. Asteroseismol., 153, 84

Kallinger, T., et al. 2008b, A\&A, 478, 497

Kallinger, T., et al. 2010, A\&A, in press (arXiv:0811.4674)

Kjeldsen, H., et al. 2005, ApJ, 635, 1281

Latham, D. W., Brown, T. M., Monet, D. G., Everett, M., Esquerdo, G. A., \& Hergenrother, C. W. 2005, BAAS, 37, 1340

Mathur, S., et al. 2010, A\&A, in press (arXiv:0912.3367)

Mazumdar, A. 2005, A\&A, 441, 1079

Merline, W. J. 1999, in ASP Conf. Ser. 185, IAU Colloq. 170, Precise Stellar Radial Velocities, ed. J. B. Hearnshaw \& C. D. Scarfe (San Francisco, CA ASP), 187

Michel, E., et al. 2008, Science, 322, 558

Miglio, A., et al. 2009, A\&A, 503, L21

Mosser, B., \& Appourchaux, T. 2009, A\&A, 508, 877

Mosser, B., Deheuvels, S., Michel, E., Thévenin, F., Dupret, M. A., Samadi, R., Barban, C., \& Goupil, M. J. 2008, A\&A, 488, 635

Otí Floranes, H., Christensen-Dalsgaard, J., \& Thompson, M. J. 2005, MNRAS, 356,671

Retter, A., Bedding, T. R., Buzasi, D. L., Kjeldsen, H., \& Kiss, L. L. 2003, ApJ, 591, L151

Roxburgh, I. W., \& Vorontsov, S. V. 2003, A\&A, 411, 215

Stello, D., Bruntt, H., Preston, H., \& Buzasi, D. 2008, ApJ, 674, L53

Stello, D., Chaplin, W. J., Basu, S., Elsworth, Y., \& Bedding, T. R. 2009, MNRAS, 400, L80

Stello, D., \& Gilliland, R. L. 2009, ApJ, 700, 949

Stello, D., Kjeldsen, H., Bedding, T. R., \& Buzasi, D. 2006, A\&A, 448, 709

Stello, D., et al. 2007, MNRAS, 377, 584

Tarrant, N. J., Chaplin, W. J., Elsworth, Y., Spreckley, S. A., \& Stevens, I. R 2007, MNRAS, 382, L48

Tassoul, M. 1980, ApJS, 43, 469

Teixeira, T. C., et al. 2009, A\&A, 494, 237 